\title{
Correction to: Oral administration of inactivated porcine epidemic diarrhea virus activate DCs in porcine Peyer's patches
}

Chen Yuan, En Zhang, Lulu Huang, Jialu Wang and Qian Yang*

\section{Correction}

The original article [1] contained an error whereby the leftmost graph in Fig. 1a mistakenly had its $\mathrm{x}$-axis labelled as 'CFST'; this has now been corrected to 'CFSE'.

Received: 12 September 2018 Accepted: 12 September 2018

Published online: 03 October 2018

\section{Reference}

1. Yuan C, et al. Oral administration of inactivated porcine epidemic diarrhea virus activate DCs in porcine Peyer's patches. BMC Vet Res. 2018;14(239) https://doi.org/10.1186/s12917-018-1568-z. 